\title{
Descobertas linguísticas para pesquisadores e aprendizes: a Linguística de Corpus e o ensino de gramática ${ }^{1}$
}

\author{
Linguistic discoveries for researchers and \\ learners: Corpus Linguistics and the teaching \\ of grammar
}

Deise Prina Dutra*

Universidade Federal de Minas Gerais - UFMG

Belo Horizonte - Minas Gerais/Brasil

Rejane Protzner Silero**

Universidade Federal de Minas Gerais - UFMG

Belo Horizonte - Minas Gerais/Brasil

\begin{abstract}
RESUMO: Este artigo discute a ocorrência de agrupamentos com a palavra for em textos argumentativos produzidos por aprendizes brasileiros de inglês de nível universitário e sugere atividades pedagógicas de descoberta de padróes linguísticos. Utilizamos ferramentas da Linguística de Corpus (LC) para análise e sugestôes de ensino de gramática. Este artigo baseia-se em dados do Corpus de Aprendizes Brasileiros do Inglês (CABrI) em comparação com outros corpora. Os resultados, que revelam o uso adequado ou não adequado de agrupamentos com verbo + for e substantivo + for, são seguidos de atividades que visam à integração da gramática com o léxico e a uma conscientização dos aprendizes sobre a frequência do uso de certos agrupamentos linguísticos.
\end{abstract}

PALAVRAS-CHAVE: corpus de aprendizes, frequência, ensino de gramática e vocabulário

\footnotetext{
${ }^{1}$ Este trabalho é um dos frutos do pós-doutorado da primeira autora na Pontifícia Universidade Católica de São Paulo sob a supervisão de Tony Berber Sardinha. Uma versão preliminar dessa pesquisa foi apresentada no VIII Encontro de Linguística de Corpus no Rio de Janeiro em 2009.

* deisepdutra@gmail.com

** rejaneprotzner@gmail.com
} 


\begin{abstract}
This article discusses the occurrences of clusters with the word for, in argumentative essays written by undergraduate Brazilian learners of English and suggests pedagogical activities to encourage the discovery of linguistic patterns. We use Corpus Linguistics (CL) tools in our analysis and pedagogical suggestions. Our research is based on data collected from the Brazilian Corpus of English Learners (Corpus de Aprendizes Brasileiros do Inglês-CABrI) in comparison with other corpora. The results, which pointed to appropriate or inappropriate use of bundles such as verb + for and noun + for, are followed by activities that aim to integrate grammar and lexis and to promote learners' awareness of frequency of use of certain linguistic bundles.
\end{abstract}

KEYWORDS: learner corpus, frequency, teaching grammar and vocabulary

\title{
Introdução
}

O tema ensino de gramática de uma segunda língua (SL) ou língua estrangeira (LE), no nosso caso, o inglês, suscita diferentes opinióes de aprendizes e professores e / ou pesquisadores. Se por um lado há aqueles que acreditam na importância do ensino da gramática para o domínio efetivo de uma língua, há outros que preferem valorizar outros aspectos da língua, visando mais ao desenvolvimento da fluência do que da acuidade. Ainda há os que advogam por um equilíbrio entre as atividades que enfatizam o desenvolvimento da fluência e da acuidade, sem a necessidade de retorno à instrução com foco puramente gramatical (RICHARDS, 2002). Antes de apresentar nossa proposta de ensino de gramática, cabe discutir em qual vertente da Linguística nos inserimos. Isso nos leva a conceituar gramática e, posteriormente, a apresentar a abordagem empirista da linguagem, utilizada pela Linguística de Corpus (LC), base para este artigo, e sua frutífera ligação com a Linguística Aplicada, especialmente com o segmento que se ocupa das questóes relativas ao ensino de línguas estrangeiras.

Optamos, neste trabalho, por nos filiar ao conceito de Linguística como uma ciência descritiva, que leva em consideração a linguagem em uso e não, necessariamente, o padrão tradicionalmente reconhecido como correto:

[...] o linguista tenta descobrir e registrar as regras que os membros de uma comunidade de linguagem seguem e não busca impor a eles outras regras (externas), ou normas do que seja correto. [...] A razão pela qual os linguistas atuais são tão insistentes sobre a distinção entre as regras descritivas e prescritivas é simplesmente porque a gramática tradicional tinha um forte caráter normativo. Os gramáticos viam 
como sua tarefa formular os padrões corretos e impô-los, se necessário, aos falantes da língua. (LYONS, 1981, p. 47-48). ${ }^{2}$

Muito embora saibamos que a área de ensino de línguas não se baseie somente na Linguística para tomar suas decisões pedagógicas, nossa opção por favorecer o caráter descritivo da Linguística pode ser explicada pelo fato de que a gramática normativa teve e, em certos contextos, ainda tem forte influência nessas decisões. Em uma das tentativas de se discutir essa influência, Westney (1994, p. 73) parte de "uma definição ampla de gramática que considera gramática como qualquer aspecto da língua possível de ser sistematicamente descrito, envolvendo não somente morfologia e sintaxe, mas também incluindo fonologia e semântica”. Esse autor, baseando-se em Leech (1988), ${ }^{3}$ afirma que a teoria gramatical e a prática pedagógica têm princípios e objetivos distintos; contudo, a gramática descritiva pode prover informaçôes à teoria ou à prática. É nesse sentido que a Linguística de Corpus (LC) pode colaborar com a Linguística Aplicada (LA), pois, além de fornecer descrições da língua em uso, dispóe de ferramentas que podem ser utilizadas no ensino de línguas. Antes de nos atermos a esses dois aspectos relevantes para este artigo, cabe explicitar melhor alguns princípios da LC.

A LC segue uma abordagem empirista da linguagem, compreendida como um sistema probabilístico. Essa abordagem "significa primazia aos dados provenientes da observação da linguagem, em geral reunidos sob a forma de um corpus" (BERBER SARDINHA, 2004, p. 30). Segundo esse mesmo autor, a tradição empirista, impulsionada por Halliday, contrapõe-se ao racionalismo na linguística, defendido por Chomsky. Para os empiristas, deve-se analisar a língua partindo-se da frequência das ocorrências reveladoras das escolhas dos

\footnotetext{
${ }^{2}$ Nossa tradução para "[...] the linguist tries to discover and record the rules to which the members of a language-community actually conform and does not seek to impose upon them other (i.e. extraneous) rules, or norms of correctness. [...] The reason why present-day linguists are so insistent about the distiction between descriptive and prescriptive rules is simply that traditional grammar was very strongly normative in character. The grammarian saw it as his task to formulate the standards of correctness and to impose these, if necessary, upon the speakers of a language." (LYONS, 1981, p. 47-48)

${ }^{3}$ Leech (1988) apud Westney (1994): LEECH, G. Varieties of English grammar: the state of the art from the grammarian's point of view. In: BALD, W-D. (Ed.). Kernprobleme der englischen Grammatik: Sprachliche Fakten und ihre Vermittlung. München: Langescheidt-Longman, 1988. p. 5-17.
} 
falantes em determinados contextos de uso. Enquanto isso, os racionalistas trabalham com as possibilidades que o sistema de uma língua coloca à disposição de seus falantes nativos. Para fins pedagógicos, é muito relevante considerar que as frequências de itens linguísticos não são aleatórias, mas, sim, dependentes do contexto (BIBER, 1988). Pesquisas recentes têm investigado, por exemplo, quais são os pacotes lexicais (lexical bundles) mais frequentes na escrita acadêmica (SIMPSON-VLACH; ELLIS, 2010), o que pode colaborar para um ensino mais efetivo àqueles que precisam ter sucesso no contexto acadêmico.

Delineamos brevemente os objetivos deste artigo, que visa exemplificar como as descriçõos da língua em uso e as ferramentas da $\mathrm{LC}^{4}$ podem ser utilizadas no ensino da gramática de língua inglesa. Mais especificamente, nosso primeiro objetivo é descrever alguns aspectos da interlíngua de aprendizes de inglês, mostrando como os erros de aprendizes dessa língua, aparentemente restritos ao uso da preposição for, podem ser tratados de maneira inovadora na abordagem baseada em corpus. Nesse sentido, essa descrição aponta para o fato de que o ensino de gramática poderá ser mais integrado ao ensino de vocabulário (CONRAD, 2000). Para atingir esse primeiro objetivo descreveremos nosso corpus de estudo (Corpus de Aprendizes de Inglês $\mathrm{CABrI}$ ), formado por redações de alunos da graduação em inglês da Universidade Federal de Minas Gerais (UFMG), em comparação com outros corpora (Contemporary Corpus of American English - COCA, Louvain Corpus of Native English Essays - LOCNESS, e o subcorpus do ICLE coletado em outras instituições que não a nossa - Br-ICLE). Nosso segundo objetivo é mostrar como o foco nas condições de uso das construções linguísticas

\footnotetext{
${ }^{4}$ Esclarecemos brevemente que a LC parte da perspectiva da língua em uso, diferenciando-se da linguística tradicional que estuda questôes estruturais das línguas (e.g. morfemas, palavas, frases etc.) (BIBER, CONRAD; REPPEN, 1998). Há autores (i.e. AARTS, 2002) que advogam que, tendo a LC o foco na língua em uso, que é distinto da ênfase da linguística teórica (que se ocupa da descrição da língua em geral), ela deve ser considerada uma disciplina. Aarts (2002) argumenta que a LC tem duas metodologias: baseada em corpus (corpus-based) e dirigida por corpus (corpusdriven). Outros autores (i.e. SCOTT; TRIBBLE, 2006, p. 4) não tratam a LC como um novo ramo de conhecimento e sim afirmam que os "métodos baseados em corpus são meramente um grupo de ferramentas e quadros". Nossa tradução para "corpus-based methods are merely a set of tools and framewoks". Neste artigo seguimos Scott e Tribble (2006), bem como Biber, Conrad e Reppen (1998), que filiam seus estudos linguísticos à abordagem baseada em corpus (corpus-based approach).
} 
(CONRAD, 2000) pode favorecer o desenvolvimento da conscientização linguística dos aprendizes. Dessa maneira, o ensino de gramática para aprendizes que necessitem atingir boa acuidade passa a ser vinculado ao verdadeiro uso da língua. Antes de nos atermos aos resultados deste estudo, apresentamos a fundamentação teórica que norteia nosso trabalho, bem como a metodologia utilizada.

\section{Fundamentação teórica}

O ensino de línguas baseado nas necessidades dos aprendizes, em alguns casos, tem ressaltado a ligação entre a forma, significado e uso (LARSENFREEMAN, 2003), considerados indissociáveis. Um dos exemplos desse enfoque seria a abordagem foco-na-forma, proposta por Long (1991), que pode ser tanto reativa quanto proativa (DOUGHTY; WILLIAMS, 1998). A abordagem reativa pressupóe que os aprendizes, ao tentarem utilizar certas estruturas linguísticas, tenham demonstrado dificuldades no seu uso apropriado, cabendo, então, por exemplo, um feedback corretivo por parte do professor (DOUGHTY; VARELLA, 1998). Já o tipo proativo da abordagem foco-na-forma implica que o professor, conhecendo seu grupo de alunos, possa prever dificuldades de aquisição de alguma estrutura e proponha atividades que ressaltem seu uso, ou seja, demonstrando como a forma é efetivamente usada em um contexto específico. Forma, significado e uso estão inequivocamente interligados, tanto na abordagem foco-na-forma reativa quanto na proativa.

Um mesmo curso pode implementar atividades reativas e proativas com sucesso, baseadas na LC, mais especificamente na proposta "Aprendizado Movido por Dados" (AMD - JOHNS, 1994), a qual se ancora na ideia de que os aprendizes de língua estrangeira devem ter a oportunidade de descobrir padrões na língua alvo a partir de análises de exemplos autênticos dessa língua (DUTRA, 2009). Esse estudo tratou das dificuldades dos aprendizes, alunos da graduação em inglês, propondo atividades preparadas com corpora online. A abordagem reativa envolveu a análise das redações produzidas pelos alunos. Esses dados mostraram, por exemplo, a necessidade dos aprendizes de desenvolverem seus conhecimentos sobre o uso apropriado do sistema de modalização do inglês. Já a abordagem proativa foi utilizada quando os alunos demonstravam interesse em aprofundar em algum tópico linguístico discutido nos textos do curso, por exemplo, o uso de porções (chunks) frequentes em inglês que têm "unidade de significados ou funções" (O'KEEFFE, MCCARTHY; CARTER, 2007, p. 46), como you know, I mean e sort of. Em ambos os 
exemplos de atividades, a gramática não era compreendida somente em relação às questôes das formas, e os aprendizes foram incentivados a fazer relações entre forma, significado e uso, ressaltando os padrões léxico-gramaticais ${ }^{5}$ que emergiam das análises feitas. Para detectar esses padrões, os aprendizes eram expostos à concordância que é "uma listagem das ocorrências de um item específico, dispostas de tal modo que a palavra de busca (aquela que se tem interesse em investigar) aparece centralizada na página (ou tela do computador)" (BERBER SARDINHA, 2004, p. 187) (ver, por exemplo, FIG. 2 deste artigo). Eles recebiam a orientação de se concentrarem nas palavras que acompanham o item linguístico em questão, seja a sua direita ou esquerda a fim de que investigassem o cotexto ${ }^{6}$ imediato, possibilitando a compreensão dos padrões léxico-gramaticais. Com a análise da concordância, destaca-se que "a palavra de busca é acompanhada do seu cotexto original, isto é, das palavras que ocorreram junto com ela no corpus." (BERBER-SARDINHA, 2004, p. 187).

Os tipos de atividades descritos acima podem propiciar o desenvolvimento linguístico e o aumento da acuidade dos aprendizes, principalmente, quando o foco no item linguístico advém das necessidades desses aprendizes. É por isso que estudos que enfocam em corpora de aprendizes (c.fBERBER SARDINHA, 2001; GRANGER, 1998; GRIES; WULFF, 2005; LÚCIO, 2006; PINTO, 2008) podem colaborar para que tenhamos melhor conhecimento sobre as dificuldades dos aprendizes, e isso pode informar a preparação de atividades pedagógicas.

Mesmo não sendo fácil compilar dados de aprendizes (ver seção de metodologia sobre a formação do subcorpus do International Corpus of

\footnotetext{
${ }^{5}$ A análise de textos autênticos possibilita a observação de que algumas estruturas tendem a ocorrer frequentemente com certas palavras, permitindo a constatação de padrōes léxico-gramaticais. "Esses padrões não são associaçōes meramente arbitrárias; mais propriamente, estruturas gramaticais específicas frequentemente ocorrem com classes lexicais restritas porque tanto as estruturas quanto as classes lexicais servem às mesmas tarefas ou funçōes comunicativas." (BIBER et al., 1999, p. 13). Nossa tradução para "These patterns are not merely arbitrary associations; rather, particular grammatical structures often occur with restricted lexical classes because both the strucutres and the lexical classes serve the same underlying communicative tasks and functions."

${ }^{6}$ Baseamo-nos em Partington (1998), Fox (1998), entre outros autores, que utilizam o termo cotexto para se referirem ao ambiente linguístico que acompanha o item linguístico em investigação. $\mathrm{O}$ termo contexto seria, então, utilizado para se referir ao ambiente situacional no qual o item é utilizado (FOX, 1998).
} 
Learner English (ICLE) com redações de aprendizes brasileiros - Br-ICLE), o número de trabalhos na área tem crescido no Brasil e em diversos países. Ponderamos, entretanto, que estudos sobre corpus de aprendizes são recentes - da década de 90 em diante. O grande destaque entre as publicações nessa área é a coletânea de Granger (1998), que abrange discussões sobre metodologia, descrição e aplicação pedagógica desse tipo de corpus. Dentre as questões abordadas, podemos exemplificar as ferramentas computacionais para a análise de corpora de aprendizes (MEUNIER, 1998), o uso de conectivos adverbiais (ALTERNBERG; TAPPER, 1998) e a instrução com foco-na-forma, bem como o aprendizado movido por dados (GRANGER; TRIBBLE, 1998). Esses estudos têm o objetivo de descrever o uso da língua-alvo pelos aprendizes (HATZITHEODOROU; MATTHEOUDAKIS, 2008), além de, em alguns casos, visar também a fazer um diagnóstico da proficiência dos aprendizes, a fim de servir como subsídio de açôes pedagógicas que utilizem os recursos da LC para propostas pedagógicas (c.f. JOHNS, 1994; MILTON, 1998).

Além disso, a compilação de corpus de aprendizes permite que conheçamos a interlíngua desses aprendizes e que façamos (a) um uso direto em sala de aula (SEIDLHOFER, 2002) com a análise das produções feitas pelos próprios alunos ou (b) um uso indireto. Nosso artigo enquadra-se na descrição da interlíngua de aprendizes de inglês para, posteriormente, propor atividades de conscientização linguística que podem fazer uso direto do corpus de aprendizes (CABrI). A área de descrição da interlíngua tem se mostrado bastante proveitosa, especialmente por contribuir com a compreensão sobre o que é a "produção de aprendizes" (learner output), que ainda se encontra um tanto quanto deficiente nos campos de pesquisa sobre o ensino de língua estrangeira. Dentre os elementos a serem analisados em um corpus de aprendizes estão seus erros. O erro, nessa perspectiva, é visto como parte do processo de aprendizagem, conforme também defendem vários autores (c.f. BERBER SARDINHA; SHEPHERD, 2008) que consideram os erros sistemáticos e passíveis de serem alvo de reflexão a respeito do desenvolvimento da interlíngua. Essa nova vertente da análise contrastiva com foco na interlíngua (GRANGER, 1998) é diferente da análise contrastiva tradicional, que comparava duas línguas, objetivando identificar as diferenças e, assim, prever erros. Os estudos que visam a investigar erros de alunos, embasados na LC, têm foco e método diferentes da Análise de Erro tradicional, priorizandose o uso de construções linguísticas para que sobreuso e subuso sejam entendidos dentro de uma perspectiva não normativa, mas, sim, descritiva da 
interlíngua. Nesse ponto, a comparação com o uso de estruturas com significados correspondentes produzidas por falantes nativos se faz necessária. Essa comparação é feita entre corpora com um grande número de dados, diferentemente do que ocorria nos estudos vinculados à Análise de Erro, cujo objeto de exame eram

coletâneas de fragmentos descontextualizados, selecionados de antemão, que exibiam certas características definidas a priori, no caso, erros, podendo se constituir em um apanhado de frases soltas ou meramente em uma lista de palavras e orações. (BERBER-SARDINHA, 2004, p. 267).

Como colocado anteriormente, a análise contrastiva da interlíngua (ACI) não é uma volta à análise contrastiva tradicional. A ACI propicia o levantamento de quais aspectos linguísticos merecem ser tratados pedagogicamente. Além disso, argumentamos que os estudos baseados em corpus podem revolucionar o ensino de gramática em três aspectos:

1. descriçõos monolíticas da gramática do inglês serão substituídas por descrições com base em registros específicos.

2. o ensino de gramática ficará mais integrado com o ensino de vocabulário.

3. a ênfase mudará da acuidade estrutural para as condições apropriadas de uso de construções gramaticais alternativas. $\left(\right.$ CONRAD, 2000, p. 549) ${ }^{7}$

De acordo com os objetivos deste artigo, propomo-nos a mostrar como Conrad pode ter razão quanto à potencialidade da LC para mudar os caminhos do ensino de gramática. Nosso estudo não prevê a descrição da gramática do inglês, mas se vale do conceito de registro para delimitar a formação de seu corpus de estudo (corpus de aprendizes constituído por redações) e também faz comparaçóes com o registro acadêmico de um corpus de referência. Este artigo pretende mostrar como o ensino de gramática, aqui representado pelo ensino

\footnotetext{
${ }^{7}$ Nossa tradução para "1. Monolithic descriptions of English grammar will be replaced by register-specific descriptions. 2. The teaching of grammar will become more integrated with the teaching or vocabulary. 3. Emphasis will shift from strucutural accuracy to the appropriate conditions of use for alternative grammatical constructions" (CONRAD, 2000, p. 549).
} 
do item linguístico for, lucra com a integração do ensino de vocabulário e, acima de tudo, o quanto o foco do ensino de gramática tem a ganhar ao promover no aluno a percepção sobre o uso de estruturas linguísticas.

Assim sendo, na próxima seção discorremos acerca das metodologias utilizadas para a compilação de nosso corpus (CABrI) e para sua análise. $\mathrm{Na}$ seção seguinte, apresentamos alguns resultados que permitirão a ligação entre a LC e o ensino de línguas a partir da análise de agrupamentos com a palavra for. Discutiremos sobre possíveis atividades voltadas para o ensino da gramática integrado ao ensino de vocabulário - e que reforce a ideia de adequação das construções linguísticas para o registro e cotexto em questão.

\section{Metodologia}

Os dados analisados são provenientes do Corpus de Aprendizes Brasileiros de Inglês (CABrI), composto de redações de alunos da graduação em Letras da UFMG (habilitação em inglês). Como este é um corpus ainda em formação, vale a pena ressaltar que, na época da análise dos dados, o corpus continha 36.187 palavras.

A maior parte dos corpora de aprendizes é de linguagem escrita, e o nosso corpus, $\mathrm{CABrI}$, está sendo formado parcialmente de acordo com os princípios e padrôes do International Corpus of Learner English (ICLE), cuja segunda versão (GRANGER; DAGNEAUX; MEUNIER; PAQUOT, 2009) contém 3.7 milhóes de palavras provenientes de 16 subcorpora (Bulgária, China, Finlândia, Suécia, Itália, entre outros). Parte do CABrI, a correspondente às redações escritas por aprendizes de nível avançado, poderá contribuir com o subcorpus de produções de aprendizes brasileiros de inglês (Br-ICLE) que fará parte do ICLE. Berber Sardinha (2001) afirmou que o Br-ICLE tinha em 200120.259 palavras (cerca de $10 \%$ do total planejado - 200 mil palavras). Até maio de 2009, o corpus tinha dobrado (40.834 palavras). Isso demonstra a dificuldade dos pesquisadores em coletar os dados necessários. No Brasil, outra grande iniciativa para a formação de corpora de aprendizes é o Corpus Multilíngue de Aprendizes (CoMAprend), constituído de redações dos alunos da graduação e dos cursos de extensão das áreas do Departamento de Letras Modernas da Universidade de São Paulo: alemão, espanhol, francês, inglês e italiano (TAGNIN; FROMM, 2008).

Os procedimentos de coleta seguiram as indicaçôes do ICLE. Primeiramente, os textos foram coletados de alunos do curso de Letras. Segundo, as redações foram escritas individualmente e fora da sala de aula. 
Terceiro, os alunos podiam consultar dicionários e gramáticas, entre outros, indicando, em um formulário sobre seu histórico escolar, quais materiais tinham sido utilizados como apoio durante a escrita da redação. Quarto, cada redação deveria ter entre 500 e 1.000 palavras, e devia ser escrita por alunos de nível avançado ou intermediário alto. Parte das redações coletadas não atendeu a esse último requisito, por terem menos de 500 palavras e por terem sido escritas por alunos de níveis iniciante e intermediário. Sendo assim, 20 redações (12.082 palavras) poderão ser integradas ao $\mathrm{Br}$-ICLE, e o restante apenas continuará como parte do CABrI, que tem como um de seus objetivos conhecer mais a fundo o nível linguístico dos alunos da graduação em inglês de nossa instituição, para que sejam realizadas intervençõos pedagógicas compatíveis com as suas necessidades linguísticas.

Os dados foram analisados segundo quatro procedimentos. Em primeiro lugar, foi feita uma análise manual para a identificação de erros, entendidos como construçóes linguísticas que se distanciam dos padrões lexico-gramaticais mais frequentes no inglês. Segundo, utilizamos o Error Tagger 1.0, disponível no site <www.corpuslg.org>, para a identificação das palavras com maior probabilidade de serem usadas erroneamente no corpus. Apesar de o Error Tagger 1.0 não possuir alta precisão (BERBER SARDINHA; SHEPHERD, 2008), resolvemos utilizá-lo, pois, por meio dele, foi possível comparar os resultados com aqueles obtidos pela análise manual e pelo programa WordSmith Tools. Terceiro, utilizamos também, em diversos momentos da análise, o programa WordSmith Tools para extrair a frequência de palavras, colocações e coligações, e analisar as linhas de concordâncias nas quais determinadas construções linguísticas ocorriam. Quarto, comparamos nossos resultados com o corpus geral COCA (Corpus of Contemporary American English) e com os corpora de aprendizes LOCNESS (Louvain Corpus of Native English Essays) e Br-ICLE (subcorpus do ICLE coletado em outras instituições que não a UFMG). Essas comparações também foram feitas em momentos distintos da pesquisa, a fim de verificar se havia sobreuso ou subuso de determinadas construções linguísticas.

O trabalho ora descrito faz comparaçóes entre corpora produzidos em situações similares. Quando analisamos as escolhas feitas pelos aprendizes de inglês, comparamo-las às produções de falantes nativos de inglês que escreveram redações argumentativas (LOCNESS) e que escreveram textos acadêmicos (e.g. ao fazermos buscas no COCA, levamos em consideração apenas as ocorrências no registro acadêmico, e não em todos os registros desse 
corpus geral de inglês). Quanto às comparações entre nosso corpus (CABrI) e o Br-ICLE, elas são justas também, uma vez que ambos os corpora são compostos de redações argumentativas produzidas por alunos de graduação com habilitação em inglês e de faixa etária semelhante. A única diferença reside no nível de proficiência de alguns aprendizes.

A partir de nossos resultados, que serão explicitados a seguir, verificamos que, principalmente para alunos de graduação em Letras - Inglês (futuros professores ou pesquisadores), a utilização de linhas de concordância provenientes das suas próprias produçóes textuais como material de atividades com foco-na-forma pode se mostrar bem proveitosa (SEIDLHOFER, 2002). Os alunos podem ter a oportunidade de reexaminar sua escrita, atentando-se aos padrões de colocações por eles utilizados e comparando com os padrões que são produzidos por falantes nativos em contextos semelhantes. Dessa forma, eles são encorajados a desenvolver uma postura mais autônoma diante do seu processo de aprendizagem, pois saberão realizar análises, fazendo uso de recursos da LC, a fim de refletir o quão próxima ou distante está sua interlíngua do que os nativos produzem.

\section{Resultados obtidos e possíveis intervenções pedagógicas}

Nesta seção, discutiremos os resultados obtidos, ressaltando o percurso de nossas tentativas de análise até chegarmos aos agrupamentos com a palavra for, quais sejam: verbo + for e substantivo + for. ${ }^{8}$ As sugestôes de atividades pedagógicas baseadas em corpus seguirão a discussão dos resultados de cada agrupamento.

A primeira análise das redações foi manual e buscou identificar erros produzidos pelos aprendizes brasileiros de inglês quando da escrita das redaçóes argumentativas. Detectamos diversos erros e, entre eles, chamou-nos a atenção o uso inapropriado de conjunções subordinadas como after, before, since, while, whereas, e também da palavra for. Ao compararmos a frequência dessas mesmas palavras no CABrI e no corpus de redações argumentativas escritas por falantes nativos (LOCNESS), constatamos que não havia diferença na frequência de uso. Esse resultado fez-nos perceber como nossa escolha dos itens linguísticos a serem analisados foi preponderantemente guiada pelo fator

\footnotetext{
${ }^{8}$ Outros agrupamentos com a palavra for constam de nosso corpus de estudo (CABrI), porém, por limite de espaço, optamos por apresentar e discutir somente verbo + for e substantivo + for.
} 
saliência, em detrimento do fator frequência. A fim de tentarmos encontrar um objeto mais definido de análise, decidimos utilizar o etiquetador de erros - Error Tagger, - versão 1.0. For apareceu entre as dez mais fortes candidatas (mais especificamente na nona posição - ver TAB. 1) a ser usada inadequadamente no corpus (CABrI). Ainda assim, o fator saliência continuou prevalecendo, uma vez que, conforme dito, já havíamos nos atentado para o uso inadequado de for em nossa análise manual.

TABELA 1

As 10 mais fortes candidatas ao erro segundo o Error Tagger 1.0

\begin{tabular}{r|l|l|r|r|r|r|r|l}
\hline & \multicolumn{1}{|c|}{ Word } & Tag & $\begin{array}{c}\text { Word } \\
\text { (prob) }\end{array}$ & $\begin{array}{c}\text { Left } \\
\text { bundle }\end{array}$ & $\begin{array}{c}\text { Right } \\
\text { bundle }\end{array}$ & Framework & Range & $\begin{array}{l}\text { Word } \\
\text { Class }\end{array}$ \\
\hline 1 & the & 0.6040 & 0.1431 & 1.00 & 1.00 & 1.00 & 0.3974 & 0.0837 \\
2 & it & 0.5321 & 0.0451 & 1.00 & 1.00 & 1.00 & 0.0641 & 0.0837 \\
3 & to & 0.5280 & 0.0354 & 1.00 & 1.00 & 1.00 & 0.0897 & 0.0431 \\
4 & a & 0.5255 & 0.0310 & 1.00 & 1.00 & 1.00 & 0.0384 & 0.0837 \\
5 & that & 0.5220 & 0.0228 & 1.00 & 1.00 & 1.00 & 0.0256 & 0.0837 \\
6 & are & 0.5176 & 0.166 & 1.00 & 1.00 & 1.00 & 0.0384 & 0.0507 \\
7 & with & 0.5142 & 0.0295 & 1.00 & 1.00 & 1.00 & 0.0128 & 0.0431 \\
8 & technology & 0.5126 & 0.0238 & 1.00 & 1.00 & 1.00 & 0.0128 & 0.0392 \\
9 & for & 0.5122 & 0.0176 & 1.00 & 1.00 & 1.00 & 0.0128 & 0.0431 \\
10 & as & 0.5100 & 0.0045 & 1.00 & 1.00 & 1.00 & 0.0128 & 0.0431 \\
\hline
\end{tabular}

Em nossa explicitação, propomos maneiras de se lançar mão dos recursos da LC no ensino da gramática a partir da descrição dos nossos resultados, os quais se baseiam em análises feitas com o programa WordSmith Tools 5.0, e em comparações com os corpora de aprendizes LOCNESS; BrICLE, e com a seção de textos acadêmicos que compõe o corpus geral COCA.

O terceiro passo da análise foi verificar no programa WordSmith Tools 5.0 o número de linhas de concordância nos quais for aparecia nos corpora CABrI; Br-ICLE; LOCNESS. Os números da TAB. 2 poderiam significar a ocorrência de um sobreuso de for pelos aprendizes brasileiros de inglês que contribuíram para a formação do CABrI? A resposta parece ser negativa, haja vista que é, no mínimo, arriscado fazer tal afirmação, partindo de números demasiado gerais. Nosso próximo passo foi analisar as diversas construções linguísticas compostas pela palavra for. Com base nesses resultados, concentramo-nos nas atividades de ensino de gramática que podem ser desenvolvidas com aprendizes que almejam ter acuidade na escrita acadêmica. 
TABELA 2

Corpora e quantidade de linhas de concordância com for

\begin{tabular}{l|c|c|c}
\hline \multicolumn{1}{c|}{ Corpus } & $\begin{array}{c}\text { Total de palavras } \\
\text { no corpus }\end{array}$ & $\begin{array}{c}\text { Linhas de concordância } \\
\text { com for }\end{array}$ & $\begin{array}{c}\text { Porcentagem das linhas } \\
\text { de concordância }\end{array}$ \\
\hline CABrI & 36.187 & 413 & $1,1412 \%$ \\
Br-ICLE & 40.834 & 335 & $0,8203 \%$ \\
LOCNESS & 324.072 & 3145 & $0,9705 \%$ \\
\hline
\end{tabular}

\section{Agrupamentos verbo + for}

Os agrupamentos verbais com for presentes no CABrI foram: work for, wait for, search for, live for, prepare + substantivo + for, consider + substantivo + for, contribute for, spread for e stand for. Atestamos que o uso dos cinco primeiros agrupamentos verbais foram apropriados. Decidimos discutir como, com recursos da LC, podemos levar os alunos a descobrirem os padrões que acompanham prepare + substantivo + for, prepare to e consider + substantivo + for.

Como o verbo prepare ocorre também com a preposição to, comparamos suas ocorrências com as ocorrências do agrupamento prepare + substantivo + for. Aparentemente, poderíamos argumentar que houve sobreuso de prepare + substantivo + for no CABrI, porém esse fato é possivelmente explicado por uma das propostas de tema de redação oferecida aos aprendizes ("Most university degrees are theoretical and do not prepare students for the real world. They are therefore of very little value."), que inclui o agrupamento em questão. A ocorrência de ambos os agrupamentos nos outros corpora de aprendizes (Br-ICLE; LOCNESS) é muito baixa ou inexistente (TAB. 3). Quanto à comparação com as ocorrências no registro acadêmico do COCA, há 585 ocorrências de prepare for e apenas 115 de prepare to, demonstrando o uso apropriado pelos aprendizes brasileiros que, de fato, utilizaram prepare for em maior número.

TABELA 3

Porcentagem de ocorrência em cada corpus

\begin{tabular}{l|c|c}
\hline Corpus & prepare + substantivo + for & Prepare + to \\
\hline CABrI & $0,0967 \%$ & $0,0359 \%$ \\
Br-ICLE & $0,0171 \%$ & $0,0171 \%$ \\
LOCNESS & $0,0022 \%$ & - \\
\hline
\end{tabular}


Por mais que os alunos tenham sido bem-sucedidos no uso desse agrupamento (prepare + substantivo + for), uma atividade possível de ser realizada teria como foco o contraste entre os tipos de colocados à direita de prepare + for e prepare + to. Exemplos do COCA (ver FIG. 1) seriam mostrados e os alunos seriam guiados a perceber que prepare for é geralmente acompanhado por um substantivo, enquanto prepare to coloca-se com um verbo. Tal atividade, aparentemente voltada apenas para a forma, pode também estender sua função e se mostrar útil para explorar o ensino de vocabulário e, até mesmo, o uso de sufixos em inglês. Na primeira linha de concordância abaixo, a palavra learning segue o agrupamento prepare for. Seria, portanto, interessante investigar se os alunos estão cientes de que o sufixo -ing é também formador de substantivos, e não só do gerúndio no inglês.

\section{prepare for}

* By following this approach children can progress and prepare for learning.

* When you prepare for trial, don't write out your questions. They'll sound stiff and...

* ...contacted the chief of police and the military to alert them to prepare for possible civil unrest;

* “...on a cold morning in March 1994 dozens of Hualapais woke up at 6:00 a.m. to prepare for a run that would take them nearly two hundred miles along the Colorado River";

* "As Dent and elections officials across the nation prepare for this November's presidential vote, they also increasingly find themselves..."

prepare to

* With the largest of Etowah's mounds in the background, archaeologists prepare to survey a section of the site

* As I prepare to descend, the three young women are already safely down

* ...the senior year experience represents a critical developmental period as undergraduate students prepare to make the difficult transition...

* ....and became convinced that city employees should prepare to take over the sales program.

* ...are some of the most influential issues facing community college leaders as they prepare to enter the next millennium.

FIGURA 1 - Dados originários do registro acadêmico do COCA 
Quanto ao segundo agrupamento verbal a ser discutido (consider + substantivo + for $)$, parece ter havido sobreuso dele no CABrI $(0,0193 \%$ de ocorrência no corpus), tendo em vista que não houve nenhuma ocorrência desse agrupamento nos outros corpora analisados.

\section{NConcordance}

1 about this issue. This essay will consider arguments for the importance of

2 about this issue. This essay will consider arguments for the supporting the

3 about this issue. This essay will consider arguments for regarding the

4 about the issue. This essay will consider arguments for the supporting the

5 involving teaching degrees, should consider, for instance, amid many other

6 about this issue. This essay will consider arguments for theoretial

7 about this issue. This essay will consider arguments for the importance of

8 people's questions. This essay will consider arguments for controlling the

\section{FIGURA 2 - Linhas de concordância com consider + substantivo + for no CABrI}

Pode ser que o sobreuso de consider + substantivo + for tenha origem na orientação que os aprendizes recebem na disciplina de escrita acadêmica da UFMG que eles cursavam na época da coleta de dados. Nesse curso, os alunos são incentivados a utilizarem porções (chunks) ou pacotes lexicais (lexical bundles) que facilitem a escrita de textos acadêmicos para que, ao realizarem um exame internacional, por exemplo, eles se sintam mais seguros e aptos a escreverem de maneira ágil. Todavia, conforme atestado, muito embora gramaticalmente correto e também compatível com o registro acadêmico, tal agrupamento não parece ter grande relevância em termos de uso. Seria o caso de se elaborar uma atividade para que os alunos investigassem a frequência e o modo de uso de outros agrupamentos frequentes no discurso acadêmico, tais como be considered as, be argued that, incluídos na Lista de Fórmulas Acadêmicas - baseada em corpora de textos acadêmicos- e que propõe a seguinte categorização funcional dessas fórmulas ou pacotes lexicais: expressões referenciais, expressões de posicionamento e expressões de organização discursiva (SIMPSON-VLACH; ELLIS, 2010). Para isso, eles teriam de ler um determinado número de textos acadêmicos, publicados em revistas de suas áreas de estudos, a fim de observar possíveis recorrências de expressões típicas desse registro com a função pragmática que eles necessitam para, posteriormente, verificar em corpora online de registro acadêmico a frequência (e.g. COCA) e os padrôes de uso desses agrupamentos. 


\section{Agrupamento substantivo + for}

Os agrupamentos substantivo + for ocorreram com baixa frequência no CABrI. Contudo, essas ocorrências ficaram salientes em nossos dados, devido, em certos casos, à sua clara adequação ou inadequação. $\mathrm{O}$ primeiro agrupamento é opportunity + for, que foi usado pelos participantes do CABrI, e também ocorreu no LOCNESS (corpus de redações produzidas falantes nativos de inglês - FIG. 3), porém não apareceu no Br-ICLE.

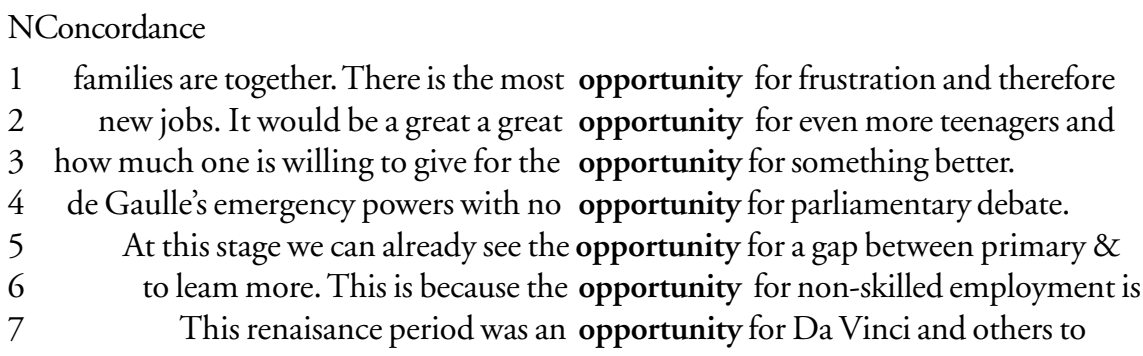

FIGURA 3 - Linhas de concordância de opportunity + for extraídas do LOCNESS

O uso de opportunity + for pelos aprendizes brasileiros se mostrou apropriado. Consideramos interessante o fato de que as linhas de concordância do CABrI (FIG. 4) apresentaram as colocaçóes provide + opportunity + for e offer + opportunity + for. No Br-ICLE e no LOCNESS, no entanto, não há ocorrências dessas colocaçóes. Decidimos, então, investigar o uso dessas ocorrências na seção do registro acadêmico do COCA, e constatamos que opportunity + for é um agrupamento muito utilizado (2.060 ocorrências). Ainda investigando o COCA, chegamos a outras constataçóes: (a) enquanto a colocação offer opportunity for obteve apenas 1 ocorrência, offer opportunities for totalizou 31 ocorrências; (b) da mesma forma, provide opportunity for obteve 13 ocorrências, enquanto provide opportunities for obteve o total de 234 ocorrências.

NConcordance

1 difficult to get. My university offers this opportunity for most of its students. We

2 are also not able to provide this opportunity for the students to put in

FIGURA 4 - Linhas de concordância de opportunity for extraídas do CABrI 
Os alunos que contribuíram para o CABrI utilizaram os agrupamentos corretamente, porém seria de grande valia que eles descobrissem que opportunities (no plural) é mais usado para ambos os agrupamentos, e que a frequência de provide opportunities for é bem maior que offer opportunities. Logo, o professor poderia, novamente, atrelar o ensino da gramática ao ensino de vocabulário, mostrando linhas de concordância de um corpus, por exemplo, do COCA, para que os alunos sejam levados a descobrir a frequência os colocados de opportunity for e o porquê do maior uso da palavra em questão (opportunity) no plural nesses agrupamentos.

Outro agrupamento formado por um substantivo + for que chamou nossa atenção foi necessity + for, não por ser este um agrupamento não adequado na língua inglesa, mas pelo fato de o seu colocado à direita ter sido um verbo no gerúndio (accomplishing). Tal estrutura (necessity + preposição + verbo no gerúndio), portanto, configura-se como um ambiente linguístico que favorece o uso de of, e não de for. Isso poderia ser explorado em uma atividade que induziria os alunos a fazerem uma consulta no registro acadêmico do COCA, com o objetivo de testar diferentes preposições até perceberem quais são as coligações adequadas e quais são suas frequências de uso, como visto brevemente na FIG. 5.

necessity for $=369$ ocorrências

(e.g. "...including the necessity for adequate corporate

governance structures"; "This is the "keystone" for lifelong learning, an absolute necessity for success in the 21 st century"; "emphasizing that Judicious Discipline is a necessity for students to feel safe, to be protected against dangers and to show respect."; "All reports agreed on the necessity for prompt removal on detection of such migration in view of the possible injury to...")

necessity of $=924$ ocorrências

(e.g. "...the fact that the necessity of developing student's critical thinking is proposed by academics..."; "Some in positions of responsibility spoke out for the necessity of change. Sister Maureen Fiedler (who now hosts the radio program Interfaith Voices..."; "For example, a spirited debate is occurring about the necessity of emphasizing evidence-based practices in behavioral health..."; "...their teachers can build on the pillars and extend them rather than face the necessity of starting over from scratch.")

FIGURA 5 - Dados extraídos do registro acadêmico do COCA 
O último agrupamento formado por um substantivo + for que comentaremos é key + for. Foi registrada uma inadequação no uso de key + for pelos participantes do $\mathrm{CABrI}$, tendo em vista que nesse corpus não há ocorrências de $k e y+$ to, enquanto no Br-ICLE e no LOCNESS só há ocorrências de $k e y+t o$, e nenhuma de key + for. Além disso, analisando os colocados à direita $(k e y+$ to + determinante + substantivo; key + to + verbo no gerúndio) em alguns exemplos aleatórios do registro acadêmico do COCA FIG. 6, percebemos que os participantes do CABrI deveriam ter optado pela preposição to, em vez de for.

key for $=72$ ocorrências

(e.g. The key for diagnosis of lupus vulgaris is the awareness of tuberculous etiology in any chronic ulcers; of these words and phrases and knowing when and how to structure this vocabulary is key for students who are wishing to understand the concepts)

key to $=1.795$ ocorrências

(e.g. and that trust among the top officials is a key to effective policymaking; In arguing for his approach Florida also contends that tolerance is key to attracting and retaining the creative class.)

\section{FIGURA 6 - Dados extraídos do registro acadêmico do COCA}

Uma atividade similar àquela proposta para necessity for poderia ser também utilizada para key for, a fim de que os aprendizes pudessem ser induzidos a verificar quais colocados são realmente usados com esse agrupamento e com qual frequência.

\section{Conclusão}

Concluímos que a palavra for, classificada como a nona palavra a ser uma forte candidata ao erro, pelo Error Tagger, nos textos do CABrI, pode tanto contribuir para formações de itens linguísticos que são usados adequada e não adequadamente pelos aprendizes brasileiros de inglês que forneceram suas redações argumentativas para esse corpus. Utilizando outras ferramentas da LC, como o programa WordSmith Tools, analisamos o CABrI, o Br-ICLE e o LOCNESS, além de acessar online o COCA, para melhor compreendermos o uso efetivo das construções analisadas. 
Dentre os agrupamentos cujo uso se aproximou daquele dos falantes nativos, estão os agrupamentos verbais preposicionados: prepare + substantivo + for, work for, wait for, search for, live for e o substantivo preposicionado opportunity for. Por outro lado, foi constatado o sobreuso de consider + substantivo + for que pode ter sua origem nas orientaçóes sobre escrita acadêmica do curso que os participantes frequentavam na época da coleta de dados. Houve também a constatação do uso inadequado de for com os verbos contribute e spread, cujos agrupamentos com a preposição to (contribute to e spread to) são muito mais frequentes nos corpora de falantes nativos.

As sugestões de atividades pedagógicas com a utilização de recursos da LC visam à descoberta dos padrôes léxico-gramaticais que ficam evidentes por meio de linhas de concordância. Primeiramente, cabe ao professor preparar atividades que induzam seus alunos a comparar suas escolhas léxico-gramaticais com aquelas feitas por falantes nativos em contexto de produção similar ao seu. Segundo, o professor deve guiá-los a descobrir os colocados mais frequentes da palavra ou agrupamento lexical em questão. Terceiro, o professor deve ressaltar que a gramática não está dissociada do léxico e que o uso de certos agrupamentos é determinado pelo contexto e cotexto. $\mathrm{O}$ acesso a corpora gratuito online é uma realidade, porém cabe ao professor preparar seus alunos utilizar esse tipo de recurso. A princípio, atividades com linhas de concordância podem ser feitas em sala de aula com material impresso, até que os aprendizes se sintam familiarizados com as descobertas que podem fazer. Posteriormente, os aprendizes podem desfrutar das ferramentas que acompanham corpora online ou até mesmo de programas como WordSmith Tools. Ressaltamos que o trabalho de conscientização linguística sugerido aqui está baseado na abordagem Aprendizado Movido por Dados (JOHNS, 1994), que pode ser de grande valia aos aprendizes que necessitem atingir um alto nível de proficiência linguística, o que é o caso daqueles que participaram da coleta de dados para nosso corpus (CABrI). 


\section{Referências bibliográficas}

AARTS, J. Does corpus linguistics exist? Soem old and new issues. In: BREIVIK. L.E.; HASSELGREN, A. (Ed.). From the COLT's mouth ... and others: Language corpora studies in honour of Anna-Brita Stenström, Amsterdam: Rodopi, 2002. p.1-16. (Republicado. In: TEUBERT, W.; KRISHNAMURTHY, R. (Ed.). Corpus Linguistics: critical concepts in Linguistics. New York: Routledge, 2007.) ALTENBERG, B.; TAPPER, M. The use of adverbial connectors in advanced Swedish learners' written English. In: GRANGER, S. (Ed.). Learner English on Computer. London \& New York: Addison Wesley Longman, 1998. p. 80-93.

BERBER SARDINHA, A. P. O corpus de aprendiz Br-ICLE. Intercâmbio, v. 10, p. 227-39, 2001.

BERBER SARDINHA, A. P. Linguistica de corpus. Barueri: Manole, 2004.

BERBER BERBER SARDINHA, A. P.; SHEPERD, T. An online system for error identification in Brazilian learner English. Anais do 8th Teaching and Language Corpora Conference. Lisboa: Associação de Estudos e de Investigação Científica do ISLA-Lisboa, 2008. p.257-262.

BIBER, D. Variation across speech and writing. Cambridge: Cambridge, 1988.

BIBER, D; CONRAD, S.; REPPEN, R. Corpus Linguistics: Investigating language structure and use. Cambridge: Cambridge, 1998.

BIBER, D.; JOHANSSON, S.; LEECH, G.; CONRAD, S.; FINEGAN, E. Longman grammar of spoken and written English. Essex:Longman, 1999.

CARTER, R.; MCCARTHY, M. Cambridge Grammar of English. Cambridge: Cambridge, 2006.

CONRAD, S. Will Corpus Linguistics revolutionize grammar teaching in the $21^{\text {st }}$ century? TESOL Quarterly, v. 34, n. 3. p. 548-560, 2000.

DOUGHTY, C.; VARELA, E. Communicative focus on form. In: DOUGHTY, C.; WILLIAMS, J. (Ed.). Focus on form in classroom second language acquisition. Cambridge: Cambridge, 1998. p. 114-138.

DOUGHTY, C.; WILLIAMS, J. (Ed.). Focus on form in classroom second language acquisition. Cambridge: Cambridge, 1998.

DUTRA, D. P. Conscientização linguística com base em corpora online. Revista Intercâmbio, v. XX, p. 79-98, 2009.

FOX, G. Using corpus data in the classroom. In: TOMLINSON, B. Materials development in language teaching. Cambridge: Cambridge, 1998. p. 25-43. 
GRANGER, S. The computer learner corpus: a versatile new source of data for SLA research. In: GRANGER, S. (Ed.). Learner English on Computer. London \& New York: Addison Wesley Longman, 1998. p. 3-18.

GRANGER, S.; TRIBBLE, C. Learner corpus data in the foreign language classroom: form-focused instruction and data-driven learning. In: GRANGER, S. (Ed.). Learner English on Computer. London \& New York: Addison Wesley Longman. 1998. p. 199-210

GRANGER, S.; DAGNEAUX, E.; MEUNIER, F. ; PAQUOT, M. International Corpus of Learner English - Version 2. Louvain-la-Neuve: UCL Presses Universitaires de Louvain, 2009

GRIES, S.; WULFF, S. Do foreign language learners also have constructions? Evidence from priming, sorting, and corpora. Annual Review of Cognitive Linguistics. v.3, p. 182-200, 2005.

HATZITHEODOROU, A.; MATTHEOUDAKIS, M. Exploring the marking of stance in argumentative essays written by EFL learners and native speakers of English. Anais do 8th Teaching and Language Corpora Conference. Lisboa: Associação de Estudos e de Investigação Científica do ISLA-Lisboa. 2008. p.133-141. JOHNS, T. From printout to handout: grammar and vocabulary teaching in the context of data-driven learning. In: ODLIN, R. (Ed.). Perspectives on Pedagogical grammar. Cambridge: Cambridge, 1994. p. 293-313.

LARSEN-FREEMAN, D. Teaching language: from grammar to grammaring. Boston: Thomson/Heinle, 2003.

LEECH, G. Varieties of English grammar: the state of the art from the grammarian's point of view. In: BALD, W-D. (Ed.). Kernprobleme der englischen Grammatik: Sprachliche Fakten und ihre Vermittlung. München: LangescheidtLongman, 1988. p. 5-17.

LÚCIO, D. D. A relexicalização de adjetivos nas redações de alunos de inglês: um estudo baseado em corpus de aprendiz. 2006. 105 f. Dissertação (Mestrado em Lingüística Aplicada) - Programa de Pós-Graduação em Lingüística Aplicada ao Ensino de Línguas, PUC-SP, São Paulo, 2006.

LYONS, J. Language and Linguistics: an introduction. Cambridge: Cambridge, 1981.

LONG, M. Focus on form: a design feature in language teaching methodology. In: de BOT, K.; GINSBERG, R.; KRAMSCH, C. (Ed.). Foreign language research in cross-cultural perspectives. Amsterdam: John Benjamins, 1991. p.39-52. 
MEUNIER, F. Computer tools for the analysis of learner corpora. In: GRANGER, S. (Ed.). Learner English on Computer. London \& New York: Addison Wesley Longman, 1998. p. 19-38.

MILTON, J. Exploiting L1 and interlanguage corpora in the design of an eletronic language learning and production environment. In: GRANGER, S. (Ed.). Learner English on Computer. London \& New York: Addison Wesley Longman, 1998. p.186-198.

O'KEEFFE, A.; MCCARTHY, M.; CARTER, R. From corpus to classroom: language use and language teaching. Cambridge: Cambridge, 2007.

PARTINGTON, A. Patterns and meanings: using corpora for English language research and teaching. Amsterdam: John Benjamins. 1998.

PINTO, M. V. O uso de things, anything, something e everything em corpora de aprendiz. 2008. 221 f. Dissertação (Mestrado em Lingüística Aplicada) Programa de Pós-Graduação em Lingüística Aplicada ao Ensino de Línguas, PUC-SP, São Paulo, 2008.

RICHARDS, J. Addressing the grammar gap in task work. In: Methodology in language teaching. Cambridge: Cambridge, 2002. p. 153-166.

SEIDLHOFER, B. Pedagogy and local learner corpora: Working with learningdriven data. In: GRANGER, S.; HUNG, J.; PETCH-TYSON, S. (Ed.). Computer learner corpora, second language acquisition and foreign language teaching. Amsterdam: Benjamins, 2002. p. 213-234.

SCOTT, M.; TRIBBLE, C. Textual patterns: key words and corpus analysis in language education. Amsterdam: John Benjamins, 2006.

SIMPSON-VLACH, R; ELLIS, N. Applied Linguistics, p. 1-26, 2010. (Publicação com acesso prévio online doi:10.1093/applin/amp058)

TAGNIN, S.; FROMM, G. CoMAprend a experiência da construção de um corpus de aprendiz para estudos. Dominio de Linguagem, 2008.

WESTNEY, P. Rules and pedagogical grammar. In: Perspectives on pedagogical grammar. ODLIN, T. (Ed.). Cambridge: Cambridge, 1994. p. 72-96.

Recebido em 4 de abril de 2010. Aprovado em 17 de maio de 2010. 